\title{
Stents Bioabsorvíveis: Já É Hora de "Absorvermos" Esse Conceito?
}

\author{
Andre Farinelli Lima Brito ${ }^{1}$, Alexandre Abizaid' ${ }^{1}$, José Ribamar Costa Jr. ${ }^{1}$
}

\section{RESUMO}

O desenvolvimento de novas estratégias para o tratamento percutâneo da doença coronariana vem sendo redirecionado para a segurança a longo prazo dos novos instrumentais, depois da marcante eficácia alcançada pelos stents farmacológicos. Dentre as tecnologias em avaliação, os stents bioabsorvíveis têm emergido como uma solução alentadora, ao trazer alguns benefícios possíveis: a) menor reação inflamatória tecidual; b) período mais curto da utilização de regime antiplaquetário duplo; e c) possibilidade futura de tratamento do vaso-alvo com enxertos venosos ou arteriais quando necessário. Alguns modelos já foram inicialmente estudados em pequenas séries clínicas, enquanto outros estão em fase avançada de investigação pré-clínica. Resultados preliminares demonstram que a menor força radial dos stents bioabsorvíveis, responsável por parte da perda luminal relacionada à retração do vaso, ainda é uma limitação dessas próteses. Dentre os stents testados, o stent polimérico com eluição de everolimus é o que no momento mostra os resultados mais encorajadores.

DESCRITORES: Implantes absorvíveis. Stents farmacológicos. Angioplastia transluminal percutânea coronária. Doença das coronárias/terapia. Ultrassonografia de intervenção.

0 5 marcantes benefícios advindos dos stents farmacológicos, com a drástica redução da proliferação neointimal e a resultante diminuição tanto da reestenose clínica como da necessidade de novas intervenções, foram parcialmente obscurecidos pela preocupação em relação à segurança desses novos dispositivos, sobretudo no seguimento a muito longo prazo $^{1-4}$.

A presença de risco aumentado de fenômenos trombóticos tardios não traria maiores desdobramen-

\footnotetext{
1 Instituto Dante Pazzanese de Cardiologia - São Paulo, SP, Brasil. Correspondência: Alexandre Abizaid. Instituto Dante Pazzanese de Cardiologia. Av. Dr. Dante Pazzanese, 500 - 14o andar - São Paulo, SP, Brasil - CEP 04012-180

E-mail: aabizaid@uol.com.br

Recebido em: 30/1/2009 • Aceito em: 28/3/2009
}

\begin{abstract}
Bioabsorbable Stents: Are We Ready to "Absorb" this Concept?

The development of new strategies for the percutaneous treatment of coronary disease has sought the long-term safety of new instruments, after the remarkable efficacy obtained by drug-eluting stents. Bioabsorbable stents have emerged as a promising solution with the following benefits: a) less tissue inflammatory reaction; b) shorter use of dual antiplatelet regimen; c) future possibility to treat target vessels with venous or arterial grafts whenever necessary. Some models were initially studied in small clinical series, while others are under pre-clinical investigation. Preliminary results show that the decreased radial force of bioabsorbable stents, responsible for part of the luminal loss related to vessel stenosis, is still a limitation of these prostheses. Of the tested stents, the polymer everolimus-eluting stent has presented the most encouraging results so far.
\end{abstract}

DESCRIPTORS: Absorbable implants. Drug-eluting stents. Angioplasty, transluminal, percutaneous coronary/methods. Coronary disease/therapy. Ultrasonography, interventional.

tos dada sua baixa incidência, não fossem tão trágicos seus desfechos ${ }^{5-7}$. Dentre as inúmeras hipóteses aventadas para explicar essa grave complicação, destacamse a marcante inibição neointimal e as reações inflamatórias locais persistentes, culminando, em última análise, em cicatrização endotelial incompleta associada ou não ao remodelamento positivo do vaso, com resultante má aposição tardia das hastes do stent e exposição crônica de hastes metálicas ao fluxo sanguíneo ${ }^{8-13}$.

Com o intuito de aumentar a segurança tardia das intervenções percutâneas, as próteses bioabsorvíveis surgiram com a proposta de resolver os problemas da atual geração de stents farmacológicos.

Vários seriam os benefícios hipotéticos dos stents bioabsorvíveis, entre eles, e o mais desejado, o de evitar o processo inflamatório tardio, reduzindo o impacto da disfunção endotelial e do remodelamento 
vascular nos vasos tratados. Permitiriam também a obtenção de imagens nos estudos não-invasivos (angiotomografia computadorizada e ressonância nuclear magnética) sem a interferência de artefatos causados pelas próteses metálicas e possibilitariam a realização da cirurgia de revascularização miocárdica quando necessária, dificultada em artérias tratadas com stents colocados em série (full metal jacket).

Dentre os vários materiais estudados para compor a prótese bioabsorvível, dois se distinguiram como os mais promissores: os polímeros e os metais.

\section{Stents bioabsorvíveis poliméricos}

Polímeros de ácido poli-L-láctico (APLL) e copolímeros de ácido poli-D-L-láctico (APDLL) têm sido utilizados em diversas áreas médicas, como ortopedia e medicina estética.

Trata-se de um material semicristalino, que, ao se solidificar, adquire um padrão misto entre cristalino e amorfo, cujas frações e orientações moleculares dependem de seus perfis térmicos e de deformação. As unidades repetitivas de polímeros de APLL/APDLL são absorvidas por meio de hidrólise, gerando ácido láctico, que, em última análise, é metabolizado no ciclo de Krebs em partículas menores que $2 \mu \mathrm{m}$ de diâmetro, as quais posteriormente acabam fagocitadas por macrófagos. O tempo para completa absorção do esqueleto polimérico gira em torno dos dois a três anos, apesar de haver absorção muito mais rápida de sua cobertura (Figura 1). Inicialmente, esses materiais estiveram associados a rea-

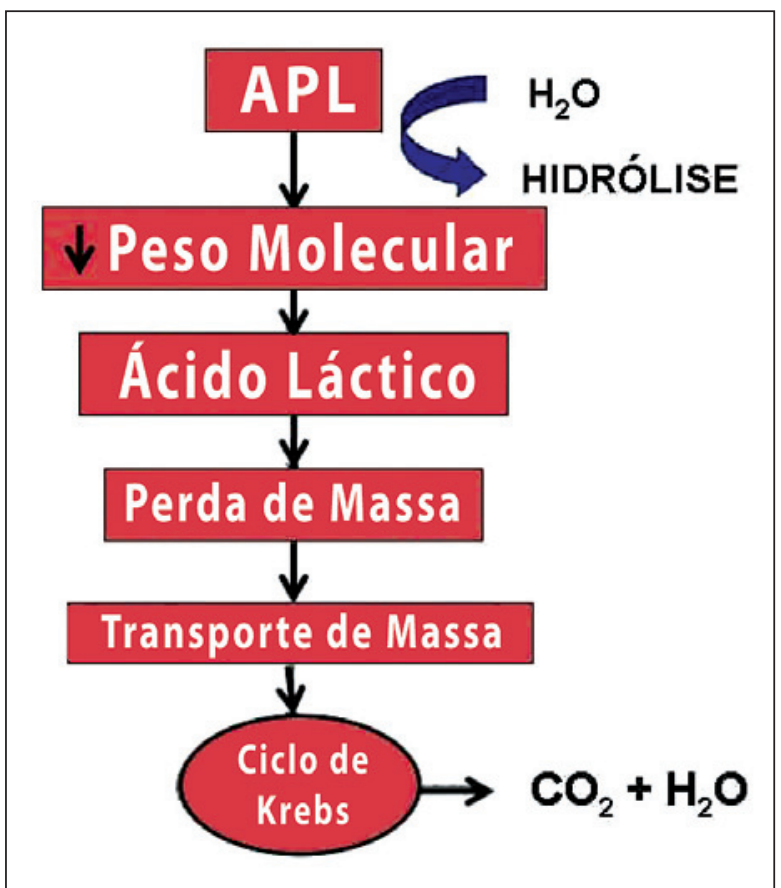

Figura 1 - Via metabólica da degradação dos polímeros de ácido poliláctico (APL). ções inflamatórias significativas, documentadas em estudos experimentais ${ }^{14}$, as quais foram bastante minimizadas com o surgimento de novas tecnologias.

O primeiro stent biodegradável polimérico considerado seguro e factível para o uso intracoronariano em humanos foi o de Igaki-Tamai (Igaki Medical Planning Co., Kyoto, Japão), cujos resultados foram inicialmente reportados em $2000^{15}$. Trata-se de um stent em forma de mola, desenvolvido com monofilamento de APLL (massa molecular de 183 kDa) balão-expansível, com desenho em ziguezague helicoidal, com 0,17 $\mathrm{mm}$ de espessura de haste e comprimento de $12 \mathrm{~mm}$ e diâmetros de 3,0 mm, 3,5 mm e $4 \mathrm{~mm}$. Quando totalmente expandido, cobre $24 \%$ da área do vaso. Possui duas marcas radiopacas de ouro em seus bordos.

Em estudo clínico inicial, 25 stents foram implantados em 19 lesões de 15 pacientes, submetidos a avaliação angiográfica e ultrassom intracoronariano um dia, três e seis meses pós-procedimento (Tabela 1). Uma peculiaridade do processo de liberação desse dispositivo foi a utilização de contraste aquecido a $80^{\circ} \mathrm{C}$ dentro do balão, com o intuito de se obter expansão adequada da prótese. A maioria das lesões (18/19 ou 94,7\%) era do tipo B2/C, conforme classificação da American Heart Association/American College of Cardiology, e o diâmetro médio de referência dos vasos tratados foi de $2,85 \mathrm{~mm}$. Obteve-se sucesso em todos os procedimentos, com retração aguda relativa [(diâmetro máximo do balão insuflado - diâmetro luminal mínimo pós-procedimento)/diâmetro máximo do balão insuflado) $x$ 100] de $22 \pm 7 \%$ aferida por angiografia coronariana quantitativa. No primeiro dia pós-procedimento, o diâmetro de estenose foi de $13 \%$ com diâmetro luminal mínimo de 2,58 mm, sem que houvesse índices de incremento adicionais da retração do stent além do observado no pós-procedimento, tanto pela angiografia coronariana quantitativa $(-3 \pm 7 \%$ ) como pelo ultrassom intracoronariano $\left(7,42 \mathrm{~mm}^{2}\right.$ vs. 7,37 $\left.\mathrm{mm}^{2}\right)$.

Após seis meses, o diâmetro médio de estenose era de $33 \%$, com perda tardia comparável à dos stents metálicos não-farmacológicos $(0,67 \mathrm{~mm})$ e aceitáveis taxas de reestenose angiográfica binária e de revascularização da lesão-alvo, ambas de 10,5\% (2/19). As análises de ultrassom intracoronariano evidenciaram aumento da área seccional transversa do stent quando comparadas às imagens pós-procedimento e às do seguimento de três meses $\left(7,42 \pm 1,51 \mathrm{~mm}^{2}\right.$ vs. 8,18 \pm $\left.2,42 \mathrm{~mm}^{2} ; \mathrm{P}<0,01\right)$, área essa que se manteve aos seis meses $\left(8,13 \pm 2,52 \mathrm{~mm}^{2}\right)$. A área de hiperplasia neointimal manteve-se inalterada dos três aos seis meses $\left(2,51 \pm 0,94 \mathrm{~mm}^{2}\right.$ para 2,50 $\left.\pm 0,65 \mathrm{~mm}^{2}\right)$. Após seis meses, ainda era possível observar hastes de stents ao ultrassom intracoronariano (Tabela 2). Não ocorreram eventos cardíacos como óbito, infarto agudo do miocárdio, revascularização ou trombose de stent, mas, em decorrência do alto perfil do dispositivo, seu uso ficou restrito às indicações extracardíacas. 
TABELA 1

Stents bioabsorvíveis

\begin{tabular}{|c|c|c|c|c|c|c|c|c|}
\hline Estudo & Tipo & $\mathbf{n}$ & Tipo do stent & Droga & Perda tardia & RB & RLA & $\begin{array}{c}\text { ECAMs } \\
(6 \text { meses })\end{array}$ \\
\hline Igaki-Tamai & Registro & 15 & Polimérico & Não & $0,67 \mathrm{~mm}$ & $6,7 \%$ & $6,7 \%$ & $6,7 \%$ \\
\hline ABSORB & Registro & 30 & Polimérico & Everolimus & $0,44 \mathrm{~mm}$ & $12 \%$ & 0 & $3,3 \% *$ \\
\hline Progress AMS & Registro & 63 & Metálico - Mg & Não & $1,08 \mathrm{~mm}$ & $47,5 \%$ & $26,7 \%+$ & $26,7 \%$ \\
\hline REVA & Registro & $30 \neq$ & Policarbonato de tirosina & Não & EA & EA & EA & EA \\
\hline BTI & Registro & $60 \neq$ & AS + polímero & Sirolimus & EA & EA & EA & EA \\
\hline
\end{tabular}

* Infarto agudo do miocárdio não-Q periprocedimento.

† Guiada por isquemia = clínica funcional.

₹ Número de pacientes previstos para inclusão, conforme protocolo dos estudos.

AS = ácido salicílico; $\mathrm{EA}=$ em andamento; ECAMs = eventos cardíacos adversos maiores; Mg = liga de magnésio; $\mathrm{n}=$ número de pacientes; $\mathrm{RB}=$ reestenose binária; $\mathrm{RLA}=$ revascularização da lesão-alvo.

TABELA 2

Comportamento da reabsorção e perda tardia

\begin{tabular}{lccccc}
\hline Estudo & $\begin{array}{c}\text { Retração aguda } \\
\text { relativa do stent } \\
\text { (ACQ)* }\end{array}$ & $\begin{array}{c}\text { Retração tardia } \\
\text { relativa do stent } \\
\text { (USIC)t }\end{array}$ & $\begin{array}{c}\text { Volume de } \\
\text { obstrução neointimal } \\
\text { (USIC) }\end{array}$ & $\begin{array}{c}\text { Perda tardia } \\
\text { (ACQ) }\end{array}$ & $\begin{array}{c}\text { Tempo de } \\
\text { absorção } \\
\text { esperado }\end{array}$ \\
\hline Igaki-Tamai & $22 \pm 7 \%$ & Ausente & $30,8 \%$ & $0,67 \mathrm{~mm}$ & $\mathrm{NR}$ \\
ABSORB & $6,9 \%$ & $11,8 \%$ & $5,54 \%$ & $0,44 \mathrm{~mm}$ & $>2 \mathrm{anos}$ \\
Progress AMS & $<5 \%$ & $\mathrm{NR}$ & $\mathrm{NR}$ & $1,08 \mathrm{~mm}$ & $4 \mathrm{meses}$ \\
\hline
\end{tabular}

* (diâmetro máximo do balão insuflado - diâmetro luminal mínimo pós-procedimento)/diâmetro máximo do balão insulflado x 100 .

+ (área média do stent pós-procedimento - área média do stent no seguimento)/área média do stent pós-procedimento x 100.

$\mathrm{ACQ}=$ angiografia coronariana quantitativa; $\mathrm{NR}=$ não relatado; USIC $=$ ultrassom intracoronariano; $\mathrm{NR}=$ não relatado.

O passo seguinte no desenvolvimento da tecnologia dos stents absorvíveis foi a incorporação de fármacos supressores de tecido neointimal, na tentativa de contrabalançar uma perda luminal tardia maior já esperada resultante da retração tardia do stent durante o processo de reabsorção.

\section{Stents poliméricos farmacológicos}

Em estudo pioneiro, Vogt et al. ${ }^{16}$ compararam, de forma randomizada, três grupos de stents implantados em modelos suínos: 1) stents bioabsorvíveis de APDLL revestidos com paclitaxel; 2) stents bioabsorvíveis de APDLL não-farmacológicos; e 3) stents metálicos nãofarmacológicos. Observaram-se, no reestudo de três semanas $(30 \pm 5 \%$ vs. $65 \pm 10 \%, P=0,021$ e vs. $53 \pm$ $6 \%, P=0,004)$ e três meses $(49 \pm 4 \%$ vs. $71 \pm 4 \%$, $P=0,029$ e vs. $68 \pm 8 \%, P=0,020)$, taxas de estenose significativamente menores no primeiro grupo quando comparado ao segundo e terceiro grupos, respectivamente.

O primeiro stent bioabsorvível farmacológico a demonstrar resultados promissores em seres humanos foi o stent liberador de everolimus BVS (Abbott Vascular, Santa Clara, Estados Unidos), avaliado por Ormiston et al. ${ }^{17}$ no estudo A bioabsorbable everolimus-eluting coronary stent system for patients with single de-novo coronary artery lesions (ABSORB) (Figura 2). Esse stent possui um esqueleto de APLL bioabsorvível revestido com uma camada rapidamente absorvível de APDLL (uma mistura de D-lactato e L-lactato), que contém e libera o fármaco antiproliferativo (Everolimus, Novartis, Basel, Suíça). O processo de bioabsorção é o mesmo descrito anteriormente. O dispositivo possui um perfil de entrega de 1,4 mm, com hastes de $150 \mu \mathrm{m}$ de espessura, e é demarcado por pontos radiopacos de metal em ambas as extremidades. A dose do everolimus foi de $98 \mu \mathrm{m}$ nos stents de $12 \mathrm{~mm}$ de comprimento e de $153 \mu \mathrm{m}$ nos stents de $18 \mathrm{~mm}$ de comprimento, com taxa de liberação de $80 \%$ em 30 dias, similar à do stent XIENCE V (Abbott Vascular, Santa Clara, Estados Unidos).

As avaliações por imagem (angiográfica, ultrassom intracoronariano e histologia virtual) e clínica foram realizadas aos seis meses e um ano, respectivamente, com taxas de perda tardia $(0,44 \mathrm{~mm})$, de reestenose angiográfica (12\%) e de revascularização da lesãoalvo (0) similares às dos stents farmacológicos utilizados na prática clínica atual (Tabela 1). 


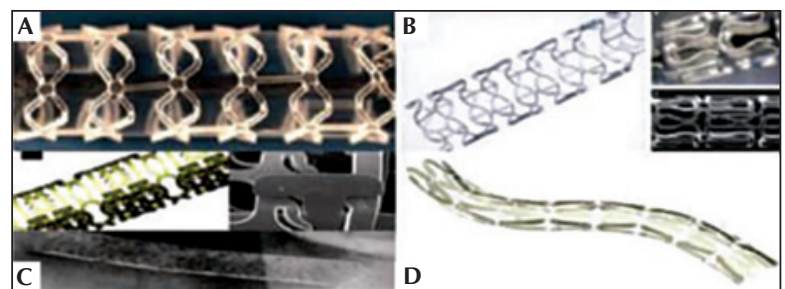

Figura 2 - Stents bioabsorvíveis. A: stent BVS (Abbott Vascular); B: stent AMS (Biotronik); C: stent REVA (REVA Medical); D: stent BTI (Bioabsorbable Therapeutics).

Resultados de dois anos de seguimento reportados recentemente demonstraram a manutenção dessas taxas de perda tardia $(0,48 \mathrm{~mm})$ e do diâmetro da estenose intrastent $(27 \%)^{18}$. A análise pelo ultrassom intracoronariano demonstrou área de hiperplasia intimal pequena $\left(0,30 \mathrm{~mm}^{2}\right)$, área do vaso mantida $\left(13,55 \mathrm{~mm}^{2}\right.$ pós-procedimento vs. $13,49 \mathrm{~mm}^{2}$ no seguimento; $P=0,98)$, e retração tardia relativa do stent de 11,8\% (6,08 $\mathrm{mm}^{2}$ pós-procedimento vs. 5,37 $\mathrm{mm}^{2}$ no seguimento; $\mathrm{P}<0,0001)$, considerada o principal mecanismo responsável pela diminuição da área luminal mínima de 24,3\% (5,11 mm² vs. 3,85 mm²; P < 0,0001) (Tabela 2). A aposição incompleta das hastes dos stents foi documentada em $6(24 \%)$ pacientes, foi resolvida em 2 e persistiu em 4 pacientes aos 180 dias. Houve 7 casos de aposição incompleta tardia, com volume da área de má aposição de $3,2 \mathrm{~mm}^{3}$, representando menos de $10 \%$ do volume do stent $\left(85 \mathrm{~mm}^{3}\right)$. A análise por meio da histologia virtual demonstrou os primeiros sinais de absorção das hastes do stent aos seis meses. Conforme a análise espectral, os tecidos localizados dentro e fora das hastes dos stents representavam material fibrótico e fibrolipídico, sem sinais de atividade inflamatória (núcleo necrótico).

Um subgrupo composto de 13 pacientes foi submetido a análise por tomografia de convergência óptica no pós-procedimento e no seguimento de seis meses. Houve aposição incompleta de $5 \%$ de suas hastes após o procedimento, que persistiram incompletas no seguimento, observando-se $1 \%$ de aposição incompleta adquirida tardia e 1\% de aposição incompleta resolvida. Em seis meses, 99\% das hastes estavam cobertas por tecido neointimal. Quanto aos desfechos clínicos, observou-se somente um (3,3\%) infarto agudo do miocárdio não-Q aos seis meses, que se manteve até os dois anos de seguimento ${ }^{18}$.

Em subanálise publicada posteriormente ${ }^{19}$, a retração tardia do stent avaliada por meio de ultrassom intracoronariano (área do stent pós-procedimento área do stent no seguimento de seis meses) foi correlacionada às características morfológicas das placas contidas nos segmentos tratados (calcificadas, fibronecróticas ou fibrocelulares). A retração tardia, absoluta e relativa, foi de $0,65 \pm 1,71 \mathrm{~mm}^{2}$ e de 7,6 $\pm 23,3 \%$, respectivamente. Placas calcificadas estiveram relacio- nadas a menores taxas de retração tardia, absoluta e relativa $\left(0,2 \pm 1,54 \mathrm{~mm}^{2}\right.$ e 1,97 $\left.\pm 22,2 \%\right)$, quando comparadas às placas fibronecróticas $\left(1,03 \pm 2,12 \mathrm{~mm}^{2} \mathrm{e}\right.$ $12,4 \pm 28 \%$, ambas as comparações com $\mathrm{P}=0,001)$ e fibrocelulares $\left(0,74 \pm 1,48 \mathrm{~mm}^{2}\right.$ e $8,9 \pm 19,8 \%$, ambas as comparações com $\mathrm{P}=0,001)$. Tal achado corrobora o conceito de que placas mais calcificadas têm menor elasticidade quando comparadas às placas fibrolipídicas, tendendo, assim, a menor retração tardia pós-dilatação percutânea.

Como a utilização de modalidades de imagem na avaliação após implante dos stents absorvíveis tem sido apontada como um de seus diferenciais, a angiotomografia coronariana computadorizada com múltiplos detectores foi testada num subgrupo de 12 pacientes do estudo ABSORB. Esse método foi utilizado três dias após o implante ${ }^{20}$, sendo comparado aos outros métodos já consagrados (angiografia coronariana quantitativa bidimensional e ultrassom intracoronariano) e também à mais moderna angiografia coronariana quantitativa tridimensional. No que tange às medidas de comprimento e diâmetro de stent e área luminal, observaram-se resultados similares entre os métodos nessa fase inicial.

\section{Stents bioabsorvíveis metálicos}

Os stents bioabsorvíveis poliméricos possuem algumas limitações: menor força radial, responsável pela retração pós-implante; taxa de bioabsorção lenta (dois a três anos), impossibilitando a retirada precoce da terapia antiplaquetária dupla; radioluscência; e alto perfil.

Buscando vencer alguns desses obstáculos, a indústria vem desenvolvendo stents bioabsorvíveis metálicos supostamente com maior força radial em comparação aos stents poliméricos, e com taxa mais rápida de bioabsorção. Até o momento, o magnésio e o ferro são as duas ligas de metal utilizadas nesse contexto, sendo o primeiro o único testado em estudo clínico.

O estudo não-randomizado multicêntrico Clinical Performance and Angiographic Results of Coronary Stenting with Absorbable Metal Stents (Progress AMS) ${ }^{21}$ avaliou a exequibilidade, a eficácia e a segurança do implante do stent AMS (Biotronik, Berlim, Alemanha), um stent bioabsorvível de liga de magnésio (Figura 2). Esse novo dispositivo, em teoria, possui desempenho similar ao dos stents de aço inoxidável, com baixa retração elástica (inferior a $8 \%$ ) e tempo de reabsorção estimado em quatro meses. Possui duas marcas radiopacas nas extremidades. Estudos experimentais demonstraram completa absorção em dois meses, associada a deposição local de cálcio e fósforo, e reendotelização completa das hastes ${ }^{22}$. Nesse estudo, foram avaliados 63 pacientes com vasos de diâmetros de 3,0 mm e $3,5 \mathrm{~mm}$ e lesões com comprimento menor que $13 \mathrm{~mm}$. Avaliações angiográfica e clínica foram realizadas em quatro e seis meses, respectivamente. Utili- 
zou-se ultrassom intracoronariano em 61 pacientes. $\mathrm{O}$ sucesso do implante e do procedimento foi atingido em $100 \%$ dos casos, confirmando a exequibilidade dessa nova tecnologia. O diâmetro de estenose residual de 12,6\% pós-procedimento corroborou a boa força radial da prótese. Entretanto, aos quatro meses, tanto a perda tardia $(1,08 \mathrm{~mm})$ como as taxas de reestenose $(47,5 \%)$ foram desapontadoras (Tabela 1 ). Não se observaram eventos cardíacos adversos como morte, infarto e trombose de stent aos seis meses.

As análises por meio do ultrassom intracoronariano demonstraram diminuída refletividade acústica das hastes aos quatro meses, apesar de poderem ainda ser identificadas. Também foi possível observar os mecanismos de perda tardia com o ultrassom intracoronariano, tendo sido resultante da diminuição do volume do vaso ( $42 \%$ da perda tardia) e da formação neointimal (responsável por $45 \%$ da perda tardia) (Tabela 2). Apesar de o estudo ter documentado a possibilidade da utilização desse dispositivo em artérias coronárias humanas, com ganho agudo significativo sem sinais de embolização distal, a expressiva retração crônica inviabilizou seu uso clínico.

Resultados também desanimadores foram obtidos com os stents AMS quando utilizados em membros inferiores ${ }^{23}$. No estudo randomizado Bioabsorbable Metal Stent Investigation in Chronic Limb Ischemia Treatment (AMS INSIGHT), com 117 pacientes (149 lesões) e comparando angioplastia com balão (57 pacientes, 75 lesões) e angioplastia com balão seguida de stent AMS (60 pacientes, 74 lesões) para lesões abaixo do joelho, a taxa de patência do grupo AMS foi inferior à do grupo controle $(31,8 \%$ vs. $58 \%$; $P=0,013)$. No entanto, manteve-se a segurança, com taxas de complicações aos 30 dias semelhantes entre os grupos $(5,4 \%$ vs. $5 \%)$.

Recentemente, Waksman et al. ${ }^{24}$ documentaram o implante de stents de ferro bioabsorvíveis em artérias coronárias porcinas. Em comparação randomizada com stents de cromo-cobalto, resultados encorajadores foram encontrados tanto no que concerne a sua eficácia como a sua segurança, de forma que avaliações preliminares em seres humanos são aguardadas no curto prazo.

\section{Comportamento endotelial pós-implante}

Tanto o stent BVS como o stent AMS tiveram o comportamento endotelial pós-implante avaliado em subgrupos. Em apresentação recente, pôde-se observar o comportamento endotelial de 7 pacientes tratados com stent BVS submetidos a teste com metergina e nitrato intracoronariano, e de 9 pacientes submetidos a teste com acetilcolina e nitrato intracoronariano $^{18}$. O primeiro grupo apresentou padrão consistente de vasoconstrição, conforme análise do diâmetro luminal médio, seguida de vasodilatação tanto nos bordos proximal $(2,7 \mathrm{~mm}, 2,49 \mathrm{~mm}$ e $2,7 \mathrm{~mm}$, respectivamente; $\mathrm{P}=0,02)$ e distal $(2,6 \mathrm{~mm}, 2,42 \mathrm{~mm}$ e
$2,67 \mathrm{~mm}$, respectivamente; $\mathrm{P}=0,03)$ como no segmento intrastent $(2,64 \mathrm{~mm}, 2,44 \mathrm{~mm}$ e 2,62 mm, respectivamente; $\mathrm{P}=0,03)$. $\mathrm{O}$ segundo grupo não demonstrou diferença no diâmetro luminal médio após acetilcolina nos três segmentos analisados, mas houve vasodilatação após o uso do nitrato em todos os segmentos: bordo proximal $(2,14 \mathrm{~mm}$ para $2,31 \mathrm{~mm}$; $\mathrm{P}=0,06)$, intrastent $(1,81 \mathrm{~mm}$ para $1,93 \mathrm{~mm} ; \mathrm{P}=0,03)$ e bordo distal $(1,76 \mathrm{~mm}$ para $2,08 \mathrm{~mm} ; \mathrm{P}=0,02)$.

Em comparação não-randomizada entre 5 pacientes do estudo Progress AMS, com 10 pacientes tratados com stents metálicos permanentes (9 farmacológicos e 1 não-farmacológico), utilizou-se o teste com dinitrato de isossorbida intracoronariano para avaliação de resposta vasodilatadora endotelial ${ }^{25}$. O segmento do vaso tratado apresentou resposta esperada ao fármaco, com aumento da área de secção transversa média, nos dois grupos sem diferença entre eles $(+13,28 \%$ vs. $+17,15 \%$; $P=0,39$ ). Quanto à análise intrastent, enquanto o grupo com stents bioabsorvíveis apresentou o mesmo padrão de resposta (aumento da área de secção transversa média), o grupo com stents metálicos permanentes mostrou resposta oposta $(+6,78 \%$ vs. $-1,3 \% ; P=0,003)$.

\section{Novos estudos e direções futuras}

Vários são os stents bioabsorvíveis em desenvolvimento no momento e duas dessas novas tecnologias estão em avaliação em nossa instituição.

\section{Stent REVA}

Esse dispositivo consiste em um stent absorvível de policarbonato de tirosina, maleável, com desenho geométrico especial de deslizamento e fechadura (possibilitando hastes mais finas) associado a um componente iodado radiopaco (Figura 2). Três centros estão desenvolvendo sua experiência first-in-man (REVA Endovascular Study of a Bioresorbable Coronary Stent - Estudo RESORB), dois na Alemanha e um no Brasil. O stent é balão-expansível, com potencial para liberação farmacológica dupla (luminal e mural), mantendo sua força radial por cerca de seis meses e planejado para ser reabsorvido em dois anos. Os desfechos primários incluem eventos cardíacos maiores em 30 dias e avaliação por meio de angiografia coronariana quantitativa (reestenose binária e perda tardia) e ultrassom intracoronariano (porcentagem de obstrução do stent) em seis meses (Figura 3).

\section{Stent BTI}

Esse stent caracteriza-se por um núcleo de ácido salicílico quimicamente incorporado a um esqueleto polimérico coberto com uma camada de salicilato que libera sirolimus (Bioabsorbable Therapeutics, Inc., BTI Polymers, Menlo Park, Estados Unidos). A liberação do antiproliferativo se dá no primeiro mês após o implante (Figura 2). 


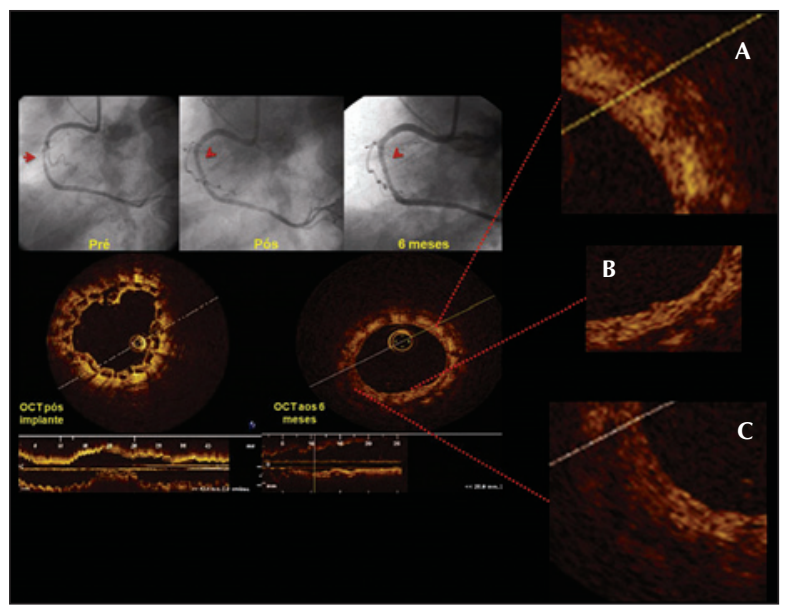

Figura 3 - Acima, cinecoronariografia pré-, pós-implante e aos seis meses do stent REVA. Abaixo, imagens da tomografia por convergência óptica em dois momentos, demonstrando única haste não aposta à parede do vaso após o implante e stent completamente incorporado à mesma parede. Detalhes à direita mostram a aparência das hastes do stent na avaliação tardia: em $\mathrm{A}$, dissolved bright box, mancha clara parcialmente visibilizada, com contornos mal definidos, sem aparência de formato de caixa, o que representa a fase intermediária de absorção do stent; em B, hiperplasia neointimal; e em C, dissolved black box, manchas negras com contornos mal definidos, frequentemente confluentes, constituindo a fase final da absorção do stent.

O Pilot Study With the IDEAL TM Bioabsorbable Coronary Stent Platform Eluting siRolimus (WHISPER) é um estudo do tipo first-in-man, multicêntrico, que pretende alocar 40 pacientes em três países (Brasil, Bélgica e Nova Zelândia). Os desfechos primários incluem avaliação com angiografia coronariana quantitativa, ultrassom intracoronariano e um subgrupo pré-especificado para análise de tomografia por convergência óptica.

\section{Desafios e possíveis soluções}

O principal desafio dessa nova tecnologia está em alcançar o equilíbrio entre suporte polimérico ótimo (com força radial e tempo de bioabsorção adequados) e tempo ideal de liberação de um antiproliferativo.

Dentre os stents poliméricos, destaca-se entre as possíveis soluções um design com distribuição mais uniforme das hastes, com menor espaço entre elas, possibilitando suporte arterial e liberação do fármaco antiproliferativo mais homogêneos e maior força radial, aplicada por mais tempo.

Quanto aos stents com ligas metálicas, um tempo mais prolongado de absorção talvez consiga diminuir parte da retração tardia, enquanto a associação de fármacos certamente reduzirá o grau de hiperplasia neointimal.

\section{CONCLUSÕES}

Enquanto novos materiais e desenhos de stents bioabsorvíveis passam pelo crivo dos testes first-in-man, permanece a busca por polímero e/ou metal capaz de sustentar uma força radial adequada e, ao mesmo tempo, ser absorvido em tempo ideal. Adicionalmente, os padrões de inflamação tecidual e biocompatibilidade associados a essa nova tecnologia deverão ser confirmados nas avaliações a longo prazo.

\section{CONFLITO DE INTERESSES}

Os autores declararam inexistência de conflito de interesses.

\section{REFERÊNCIAS BIBLIOGRÁFICAS}

1. Morice MC, Serruys PW, Sousa JE, Fajadet J, Ban Hayashi E, Perin M, et al.; RAVEL Study Group. Randomized study with the sirolimus-coated Bx Velocity balloon-expandable stent in the treatment of patients with de novo native coronary artery lesions. A randomized comparison of a sirolimus-eluting stent with a standard stent for coronary revascularization. $\mathrm{N}$ Engl J Med. 2002;346(23):1773-80.

2. Moses JW, Leon MB, Popma JJ, Fitzgerald PJ, Holmes DR, O'Shaughnessy C, et al.; SIRIUS Investigators. Sirolimus-eluting stents versus standard stents in patients with stenosis in a native coronary artery. N Engl J Med. 2003;349(14):1315-23.

3. Stone GW, Ellis SG, Cox DA, Hermiller J, O'Shaughnessy C, Mann JT, et al.; TAXUS-IV Investigators. A polymer-based, paclitaxel-eluting stent in patients with coronary artery disease. N Engl J Med. 2004;350(3):221-31.

4. Babapulle MN, Joseph L, Bélisle P, Brophy JM, Eisenberg MJ. A hierarchical Bayesian meta-analysis of randomised clinical trials of drug-eluting stents. Lancet. 2004;364(9434):583-91.

5. Kastrati A, Mehilli J, Pache J, Kaiser C, Valgimigli M, Kelbaek $\mathrm{H}$, et al. Analysis of 14 trials comparing sirolimuseluting stents with bare-metal stents. N Engl J Med. 2007; 356(10):1030-9.

6. Stone GW, Moses JW, Ellis SG, Schofer J, Dawkins KD, Morice MC, et al. Safety and efficacy of sirolimus and paclitaxel-eluting coronary stents. N Engl J Med. 2007;356(10): 998-1008.

7. Mauri L, Hsieh WH, Massaro JM, Ho KK, D'Agostino R, Cutlip DE. Stent thrombosis in randomized clinical trials of drug-eluting stents. N Engl J Med. 2007;356(10):1020-9.

8. Cook S, Wenaweser P, Togni M, Billinger M, Morger C, Seiler $C$, et al. Incomplete stent apposition and very late stent thrombosis after drug-eluting stent implantation. Circulation. 2007;115(18):2426-34.

9. Siqueira DA, Abizaid AA, Costa JR, Feres F, Mattos LA, Staico $R$, et al. Late incomplete apposition after drug-eluting stent implantation: incidence and potential for adverse clinical outcomes. Eur Heart J. 2007;28(11):1304-9.

10. Hassan AK, Bergheanu SC, Stijnen T, van der Hoeven BL, Snoep JD, Plevier JW, et al. Late stent malapposition risk is higher after drug-eluting stent compared with bare-metal stent implantation and associates with late stent thrombosis. Eur Heart J. In press 2009.

11. Lüscher TF, Steffel J, Eberli FR, Joner M, Nakazawa G, Tanner FC, et al. Drug-eluting stent and coronary thrombosis: biological mechanisms and clinical implications. Circulation. 2007;115(8):1051-8.

12. Finn AV, Nakazawa G, Joner M, Kolodgie FD, Mont EK, Gold HK, et al. Vascular responses to drug eluting stents: importance of delayed healing. Arterioscler Thromb Vasc Biol. 2007;27(7):1500-10.

13. Nebeker JR, Virmani R, Bennett CL, Hoffman JM, Samore 
$\mathrm{MH}$, Alvarez J, et al. Hypersensitivity cases associated with drug-eluting coronary stents: a review of available cases from the Research on Adverse Drug Events and Reports (RADAR) project. J Am Coll Cardiol. 2006;47(1):175-81.

14. van der Giessen WJ, Lincoff AM, Schwartz RS, van Beusekom HM, Serruys PW, Holmed DR Jr, et al. Marked inflammatory sequelae to implantation of biodegradable and nonbiodegradable polymers in porcine coronary artery. Circulation. 1996;94(7):1690-7.

15. Tamai H, Igaki K, Kyo E, Kosuga K, Kawashima A, Matsui $S$, et al. Initial and 6-month results of biodegradable polyI-lactic acid coronary stents in humans. Circulation. 2000;102(4): 399-404.

16. Vogt F, Stein A, Rettemeier G, Krott N, Hoffmann R, von Dahl J, et al. Long-term assessment of a novel biodegradable paclitaxel-eluting coronary polylactide stent. Eur Heart J. 2004;25(15):1330-40.

17. Ormiston JA, Serruys PW, Regar E, Dudek D, Thuesen L, Webster MW, et al. A bioabsorbable everolimus-eluting coronary stent system for patients with single de-novo coronary artery lesions (ABSORB): a prospective open-label trial. Lancet. 2008;371(9616):899-907.

18. Serruys PW, Onuma Y. My next "rrosy prophecy": bioabsorbable stents will become a "breakthrough" coronary technology rational, challenges and likely solutions! In: XX Transcatheter Cardiovascular Therapeutics. Washington; 2008.

19. Tanimoto S, Bruining N, van Domburg RT, Rotger D, Radeva $\mathrm{P}$, Ligthart JM, et al. Late stent recoil of the bioabsorbable everolimus-eluting coronary stent and its relationship with plaque morphology. J Am Coll Cardiol. 2008;52(20):1616-20.

20. Bruining N, Tanimoto S, Otsuka M, Weustink A, Ligthart J, de Winter S, et al. Quantitative multi-modality imaging analysis of a bioabsorbable poly-L-lactic acid stent design in the acute phase: a comparison between 2- and 3D-QCA, QCU and QMSCT-CA. Eurolntervention. 2008;4(2):285-91.

21. Erbel R, Di Mario C, Bartunek J, Bonnier J, de Bruyne B, Eberli FR, et al. Temporary scaffolding of coronary arteries with bioabsorbable magnesium stents: a prospective, non-randomised multicentre trial. Lancet. 2007;369(9576):1869-75.

22. Waksman R, Pakala R, Kuchulakanti PK, Baffour R, Hellinga D, Seabron R, et al. Safety and efficacy of bioabsorbable magnesium alloy stents in porcine coronary arteries. Catheter Cardiovasc Interv. 2006,68(4):607-17.

23. Bosiers $M$; on Behalf of the AMS INSIGHT Investigators. AMS INSIGHT - absorbable metal stent implantation for treatment of below-the-knee critical limb ischemia: 6-month analysis. Cardiovasc Intervent Radiol. 2008. Disponível em: http://www.springerlink.com/content/x17534087166w88m/ fulltext.pdf.

24. Waksman R, Pakala R, Baffour R, Seabron R, Hellinga D, Tio FO. Short-term effects of biocorrodible iron stents in porcine coronary arteries. J Interv Cardiol. 2008;21(1):15-20.

25. Ghimire G, Spiro J, Kharbanda R, Roughton M, Barlis P, Mason $M$, et al. Initial evidence for the return of coronary vasoreactivity following the absorption of bioabsorbable magnesium alloy coronary stents. Eurolntervention. 2008;4(4):481-4. 